\title{
Template matching as a tool for annotation of tomograms of stained biological structures
}

\author{
Misjaël N. Lebbink ${ }^{\mathrm{a}, *}$, Willie J.C. Geerts ${ }^{\mathrm{a}}$, Theo P. van der Krift ${ }^{\mathrm{a}}$, Maurice Bouwhuis ${ }^{\mathrm{b}}$, \\ Louis O. Hertzberger ${ }^{c}$, Arie J. Verkleij ${ }^{\text {a }}$, Abraham J. Koster ${ }^{\mathrm{d}, *}$ \\ ${ }^{a}$ Cellular Architecture and Dynamics, Utrecht University, The Netherlands \\ ${ }^{\mathrm{b}}$ SARA Computing and Networking Services, Amsterdam, The Netherlands \\ ${ }^{c}$ Department of Computer Science, University of Amsterdam, The Netherlands \\ ${ }^{\mathrm{d}}$ Molecular Cell Biology-Electron Microscopy, Leiden University Medical Center, The Netherlands
}

Received 13 September 2006; received in revised form 5 December 2006; accepted 6 December 2006 Available online 22 December 2006

\begin{abstract}
In recent years, electron tomography has improved our three-dimensional (3D) insight in the structural architecture of cells and organelles. For studies that involve the 3D imaging of stained sections, manual annotation of tomographic data has been an important method to help understand the overall 3D morphology of cellular compartments. Here, we postulate that template matching can provide a tool for more objective annotation and contouring of cellular structures. Also, this technique can extract information hitherto unharvested in tomographic studies. To evaluate the performance of template matching on tomograms of stained sections, we generated several templates representing a piece of microtubule or patches of membranes of different staining-thicknesses. These templates were matched to tomograms of stained electron microscopy sections. Both microtubules and ER-Golgi membranes could be detected using this method. By matching cuboids of different thicknesses, we were able to distinguish between coated and non-coated endosomal membrane-domains. Finally, heterogeneity in staining-thickness of endosomes could be observed. Template matching can be a useful addition to existing annotation-methods, and provide additional insights in cellular architecture.
\end{abstract}

(C) 2007 Elsevier Inc. All rights reserved.

Keywords: Electron microscopy; TEM tomography; Image processing; Biological membranes; Microtubules

\section{Introduction}

Electron tomography is an imaging technique, using transmission electron microscopy (TEM), to explore the structural architecture of cells and organelles in three dimensions (3D). This is achieved by recording two-dimensional (2D) images of a specimen at different tilt angles. A 3D reconstruction (tomogram) is calculated from the $2 \mathrm{D}$ projections by a computational method (e.g. back-projection), resulting in a digital $3 \mathrm{D}$ representation of the specimen. Typically, for those applications where stained sections are

\footnotetext{
* Corresponding authors. Fax: +31 302513655 (M.N. Lebbink), +31 71 5268270 (A.J. Koster).

E-mail addresses: M.N.Lebbink@bio.uu.nl (M.N. Lebbink), A.J.Koster @1umc.nl (A.J. Koster).
}

imaged, digital slices with a Z-resolution of 2 to $5 \mathrm{~nm}$ are visualized (Kremer et al., 1996; Koster et al., 1997). These digital slices are then used to generate $3 \mathrm{D}$ contours of the structure. Electron tomography has become an indispensable tool in morphological studies concerning 3D structural analysis and membrane connectivity; providing additional insight into cellular organization (Murk et al., 2004; Mogelsvang et al., 2004; Lučić et al., 2005; Frey et al., 2006).

Among others, the resolution in a tomogram depends on the number of angles at which 2D projections were taken. This number is limited by several technical restrictions. First, at high tilt angles, e.g. higher than $65^{\circ}$, the sample holder and the thickness of the sample will provide a limit. Second, exposure to the electron beam has to be limited to prevent specimen changes (Bennett, 1974; Egerton et al., 2004). The missing projections at high tilt angles will result 
in a direction-dependent loss of resolution in the tomogram (McEwen et al., 1995; Koster et al., 1997). This artefact (due to missing wedge) can be reduced by combining two perpendicular tomograms of the same area, resulting in clearer and sharper cellular structures with more isotropic resolution (Penczek et al., 1995; Mastronarde, 1997).

Electron tomograms show an overwhelming amount of structural detail. To better understand a particular arrangement within a tomogram, a segmentation step is performed to highlight the structure of interest and to remove unrelated information. In electron tomography of stained sections this is often done in a manual fashion by computer assisted drawing of membrane-structures in each digital slice throughout the tomogram. These traced contours are combined into a 3D model used for further analysis. Due to the manual nature of this annotation-step, some degree of subjectivity cannot be prevented.

During the last decade several powerful methods became available for the 3D segmentation of structures within tomograms. Some of these methods focused on improving the signal-to-noise ratio within tomograms (e.g. anisotropic diffusion filtering by Frangakis and Hegerl, 2001), on careful analysis of texture and contrast (e.g. watershed by Volkmann, 2002) or on development of wavelet filtering (Frangakis et al., 2001; Moss et al., 2005). A limitation of these approaches is that no special means are taken to avoid misinterpretation, or mis-segmentation, due to the missing wedge in tomograms, a characteristic phenomenon in electron tomography.

Another approach to highlight structures of interest is template matching, as described by Böhm and Frangakis (Böhm et al., 2000; Frangakis et al., 2002). With template matching, a small 3D volume containing the structure (the template) to be detected is matched to every location within the tomographic reconstruction. The computer determines the maximal cross-correlation value based upon the similarity between the template and tomogram, at every location within the tomogram and for all (predefined) angular orientations. The cross-correlation coefficient indicates how similar the matched template and the structure in the tomogram are. This approach has proven to work well for cryo-applications to localize macromolecular complexes in cells, or to localize, and average, repetitive features in parts of tomograms with templates obtained with higher resolution techniques.

We propose to use template matching as a reasonably objective method to detect and annotate a wide range of cellular structures in cryo-fixed, freeze substituted and plastic embedded sections. It should be noted that while the matching is an automated and objective process, the determination of the optimal threshold of correlation still occurs manually on visual criteria. The optimal threshold of correlation is defined as the lowest cross-correlation value at which no false positive matches could be observed (and thus a maximum of exclusively correctly matched structures was segmented). Segmentation based on template matching allows relatively low resolution morphological information, such as membranebound organelles, to be contoured and annotated in a semi- automatic fashion. In addition, by zooming in at the automatically annotated structures, morphological features that were possibly otherwise missed by the human eye, due to the overwhelming amount of stained features in a cell section, might become available. We have chosen to create simplified artificial templates to segment the morphological feature of interest computationally. An advantage of these simple templates is that, depending on the feature of interest, templates can be generated based upon general 3D shapes like cylinders, cubes, spheres, or cuboids. In this manuscript, for the relatively lowresolution requirements of the template-matching application, we built cylinders to mimic microtubules and cuboids (solid rectangular boxes) to mimic patches of membrane. The computer generated templates allow for simple controlled manipulation of a single property of the structure contained therein. That way, this property can be investigated by comparing how well each template fits. For this we compared the matching patterns of the different cross-correlations at the same manually defined threshold. To exemplify this, we used cuboid-shaped templates of different voxel-thicknesses to investigate inhomogenously stained membranes.

Variations in membrane staining may indicate differences in localization of receptor proteins and signal transduction molecules. For instance, in stained sections, membrane-coats, such as clathrin, usually appear darker and thicker than (surrounding) non-coated membranes (Ladinsky et al., 1999). Furthermore, biochemical studies have shown membrane composition to be inhomogeneous throughout the cell. For example, throughout the secretory pathway, the concentrations of cholesterol per phospholipid and especially sphingomyelin increase (Yunghans et al., 1970; Zambrano et al., 1975; Moreau and Cassagne, 1994), and thereby induce thickening of the membrane (Nezil and Bloom, 1992; Smondyrev and Berkowitz, 1999). In addition, lipid-composition might cause a depletion or accumulation of certain proteins in membrane domains (Simons and Ikonen, 1997; Killian, 1998). Each of these events can have an influence on staining efficiency/capability; indirectly indicating differences in biological functions. It must however be noted, that staining can also be the source for artefacts and misinterpretation. Therefore, it can be difficult - and often impossible - to compare different sections if the correct controls are not included; even when they are obtained under the same experimental conditions. Even a comparison between two (distant) regions of the same section should be avoided because of an inhomogeneous penetration of the staining liquids. Nevertheless, if differences in staining between neighbouring regions of the same membrane can be observed, then this can be considered an indication for differences in biological composition.

\section{Material and methods}

\subsection{Sample preparation and electron tomography}

Human dendritic cells were grown and prepared as described by Murk et al. (2003). TEM tomography was 
performed with a Tecnai 20 microscope $(200 \mathrm{kV})$ by taking two perpendicular tilt series of angular projections starting at $-65^{\circ}$ with an increment of $1^{\circ}$. These series were reconstructed and combined into a double tilt electron tomographic reconstruction using IMOD (Kremer et al., 1996).The areas of interest were cropped from the double tilted tomographic images. The template matching approach was evaluated on three tomograms. The sizes of the tomograms were $528 \times 256 \times 544$ voxels with a voxelsize of $1.34 \times 1.34 \times 1.34 \mathrm{~nm}, 1024 \times 88 \times 1024$ voxels with a voxel-size of $1.68 \times 1.68 \times 1.68 \mathrm{~nm}$, and $1024 \times 128 \times 1024$ voxels with a voxel-size of $1.68 \times 1.68 \times 1.68 \mathrm{~nm}$; respectively, for the endosome, and two tomograms containing cellular structures including the ER and Golgi-apparatus. The tomogram of the endosome was binned to dimensions of $264 \times 128 \times 272$ voxels.

\subsection{Template matching}

For the template matching algorithm we used the Omnimatch program, kindly provided by the Baumeister group at the Max-Planck-Institute for Biochemistry, Martinsried. The OmniMatch software was implemented on a computer-cluster at the SARA high performance computing facilities in Amsterdam, the Netherlands. Matlab (Mathworks Inc.) was used in combination with the TOM-toolbox scripts (Nickell et al., 2005) to create the templates, masks, and convert the datatypes. Amira (TGS) was used for volume visualization and surface rendering.

First, to be compatible with Omnimatch software, the grey-scale tomograms as produced by IMOD were inverted, such that the stained membranes appear bright on a dark background. Two types of templates (with file dimensions of $32 \times 32 \times 32$ voxels) were created with MatLab and the TOM-toolbox. The tubular template had an inner-radius of three voxels, an outer-radius of six voxels, and a length of 32 voxels (Fig. 1A and B). The cuboids with dimensions $X$ and $Z$ equal to the template size, and even thicknesses in $Y$-dimension ranging from 4 to 18 voxels

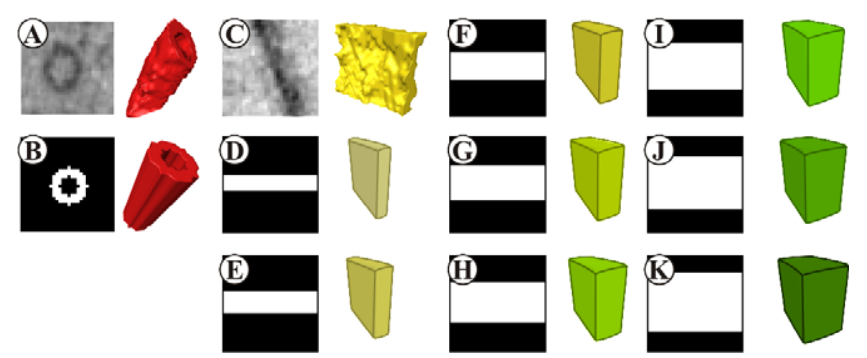

Fig. 1. Templates used for matching. In the tomograms, microtubules (A) and membrane $(\mathrm{C})$ were matched using computationally generated $3 \mathrm{D}$ templates (B; D-K). As a template for the microtubules, an open cylinder with an inner radius of three voxels and an outer radius of six voxels was created (B). For the membrane, a cuboid-shaped templates were created (D-K) with a thickness of $4,6,8,10,12,14,16$, and 18 voxels. The pseudocolours in the $3 \mathrm{D}$ representations of the templates are assigned from light yellow for a low template thickness to dark green for a high template thickness.
(Fig. 1C-K). The structure was given value 1 (white) while the background was value 0 (black). Finally, masks were created to define the area containing the structure in the template. The masks were binary $3 \mathrm{D}$ images which indicate the area within the template that contains the structure of interest. Only those voxels in the template that correspond to a voxel in the mask with a value of 1 are taken into account for the correlation process; while those that correspond to a voxels of value 0 are excluded. A spherical mask (value $=1$ ) with a radius of 14 voxels was created on a black background (value $=0$ ). Dedicated masks were created for the cuboid templates based upon the thickness in $Y$ by adding two additional voxels (containing background values in the template) on either side. The thicknesses of these masks range from 8 to 22 voxels. Finally, the point-spread function $(\mathrm{PSF})$ was described as a single white voxel $($ value $=1$ ) at position $(1,1,1)$. The PSF describes the distortion of a single pixel due to acquisition and processing of the image, and can be used to take missing wedge/pyramid-artefacts into account by distorting the template prior to the crosscorrelation-step. A single white voxel mimics an ideal microscope and perfect data collection in which no distortion takes place, and no wedge-artefacts are present.

The Euler angles, as defined in the TOM toolbox (Nickell et al., 2005), over which the template was rotated were $180^{\circ}$ over psi $(\psi)$ and theta $(\theta)$ with an increment of $5^{\circ}$; beyond these values repetition of the template occurs. Due to symmetry, rotation over phi $(\varphi)$ was not necessary, since the

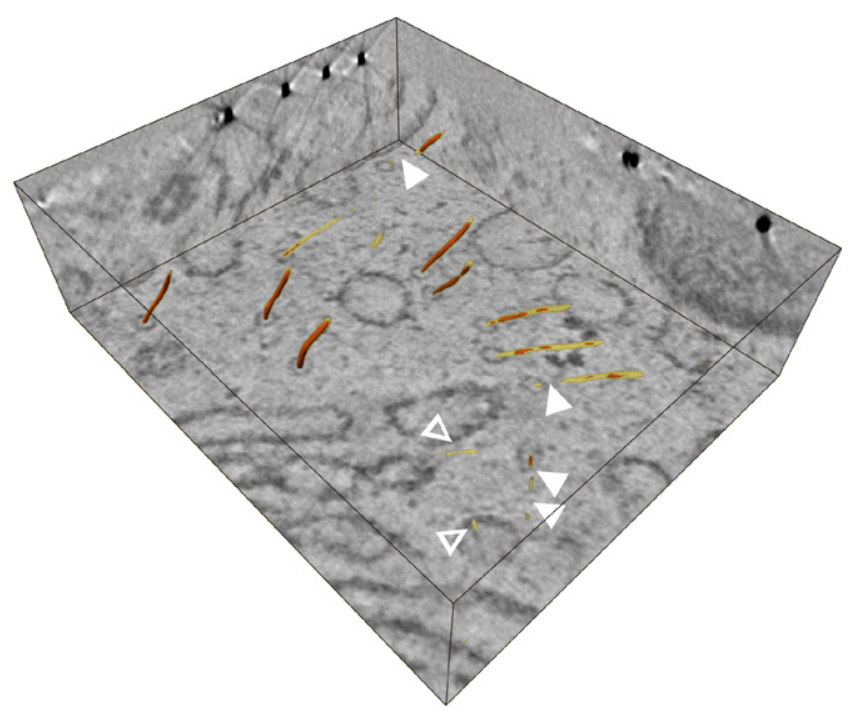

Fig. 2. Localization and annotation of microtubules. Microtubules were localized using template matching with a tubular template and a spherical mask. Template matching localizes the structure of interest: a brighter peak in the correlation file indicates when the template is present in the tomogram. This peak is visualized by setting a grey-value threshold. Red shows the regions of microtubules that were identified at a threshold value of 0.485 (the optimal threshold of correlation) and the transparent yellow lines show the regions of the microtubules that were identified at a relatively low threshold ( $\approx 20 \%$ below the optimal threshold of correlation). Lowering the threshold leads to less false negatives, and thus to more complete tubules; even though some holes still exist (closed arrowheads). False positives appear if the threshold is set too low (open arrowheads), falsely indicating the presence of a microtubule at this location. 

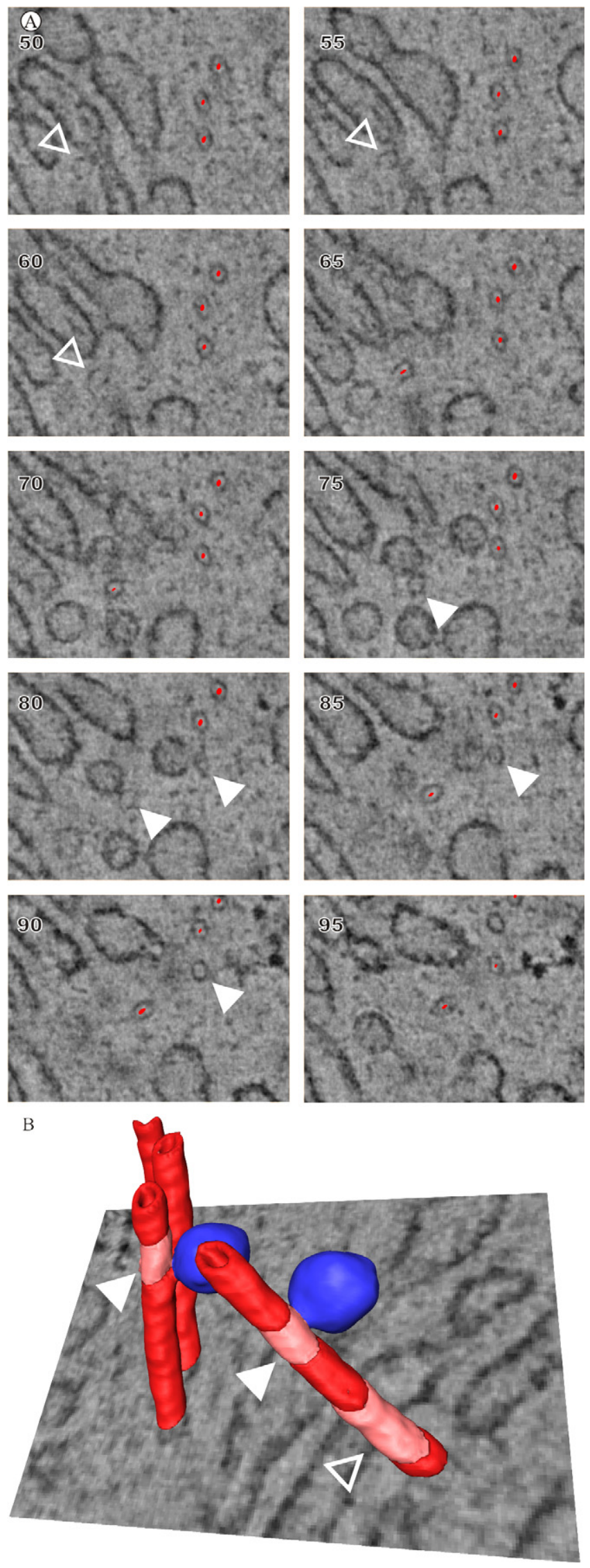

shape of the templates was such that rotation over this axis would not produce a new image. The dimension of the Fourier transform was set to the smallest dimension in the tomographic volume. The different calculations took approximately 20,70 , and $90 \mathrm{~min}$, respectively, for each volume, using 54 parallel CPUs.

\subsection{Data visualization}

The outcome of the template matching procedure is a $3 \mathrm{D}$ volume with cross-correlation values. For the segmentation and contouring approach, these cross correlation values (ranging from -1 to +1 ) were visualized by surface rendering, using Amira. To reduce the requirements on in-core computer memory, the 16 bit cross-correlation values as produced by Omnimatch were scaled and converted to 8 bit images. By varying the grey-value threshold during surface rending it is possible to visualize the location of a matched structure. To describe the performance of template matching for the purpose of contouring, we introduce the terms 'false positive' and 'false negative' annotation. With false positive annotation is meant that, at a particular threshold, the surface-rendered cross correlation values show features which should not be shown from a cell-biological point of view. For instance, false positive annotated structures could be elongated parts of membranes while it is attempted to annotate only microtubules. With false negative annotation is meant that parts of a particular organelle that, based upon visual inspection of the tomogram by a cellbiologist, should have been annotated but was not.

\section{Results and discussion}

\subsection{Automatic detection of microtubules in TEM-images}

At relatively low resolution, $\sim 5 \mathrm{~nm}$, microtubules share a very homogeneous, tubular, shape. In electron tomographic reconstructions of stained sections microtubules are often abundant, and relatively easy to detect and recognize upon visual inspection. Moreover, microtubules are involved in many cell biological processes and automated annotation of these structures in tomograms can contribute to further understanding of cell biological events as it can provide a skeleton of annotated (known) structures. These

Fig. 3. Areas with false negatives on microtubules. The top panel (A) shows a series of digital slices through a tomogram. Localization results are shown in red with a threshold of $0.4(\approx 20 \%$ below the optimal threshold of correlation). Vesicles that appear to be attached to the microtubules gave rise to false negatives (closed arrowheads). Similarly, membranes of the Golgi-apparatus in proximity of the microtubules result in false negatives of the matched microtubule (open arrowheads). These results are visualized in a manually annotated $3 \mathrm{D}$ model (B) where red shows the regions that were localized using template matching, pink are the false negatives that the procedure missed, and in blue vesicles are shown. The arrowheads show the same regions as presented in the top panel. 
arguments make microtubules suitable for evaluating the template matching procedure.

In Fig. 2 annotated microtubules are shown. At a first glance, most microtubules appear to be detected correctly. On closer examination, we observed that (even at a low correlation-threshold when false positives appear (open arrowheads)) holes are present in some of the detected microtubules (closed arrowheads). When analysing the tomographic regions that were missed using template matching, we noticed that these holes revealed biological occurrences easily overlooked by manual annotation. For instance, as shown in Fig. 3, in several of these regions, vesicles (Fig. 3A; closed arrowhead, Fig. 3B) or other membranous structures (Fig. 3A; open arrowhead) associated with the microtubules could be observed. Fig. 4 shows correctly annotated microtubules, together with a false positive annotated structure; membrane tubulation (Fig. 4; closed arrowhead). While misinterpreted from a biological point of view, the template correctly annotated a short cylindershaped feature.

Based upon these observations, we conclude that template matching can be used as an additional tool to detect structures like microtubules within a TEM-reconstruction containing stained cellular structures. Furthermore, we showed that while localization and morphology can be examined based on where the template fits in the tomogram, false negatives can indicate equally significant information from a cell biological point of view.

\subsection{Visualization of membranes in the ER and Golgi- apparatus}

Membranes are extensively modelled features in electron tomograms of stained sections. While at a 5-nm resolution
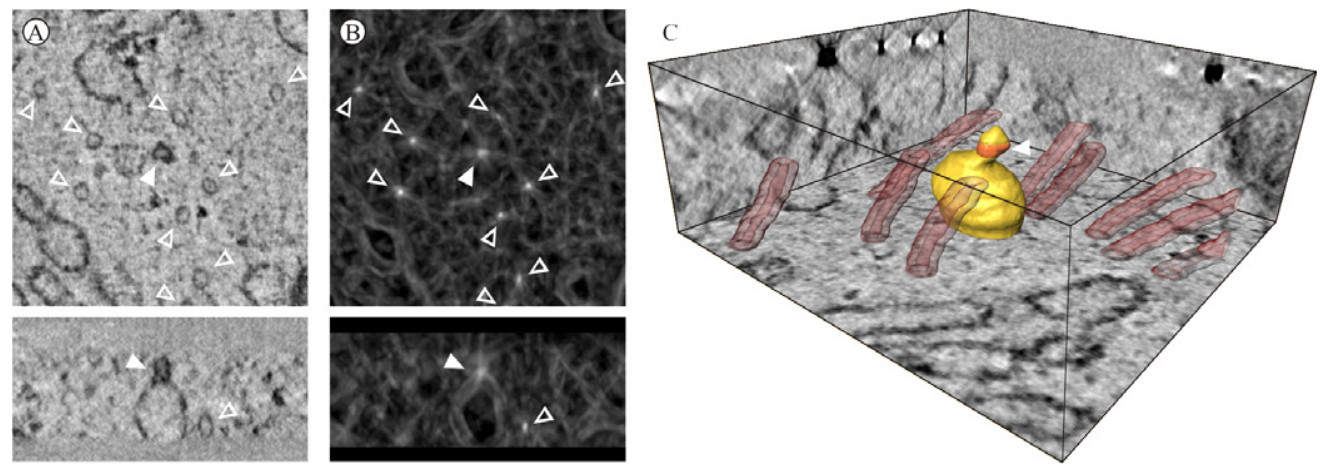

Fig. 4. Areas with false positives on microtubules. Tubulation of a membrane (A; closed arrowheads) resembles the microtuble-template. As a consequence, a bright correlation-peak could be observed at that location in the tomogram (B; closed arrowhead) along with the correlation-peaks of the microtubules (open arrowheads). This tubulation was visualized in a 3D model by manual annotation. The orange region (C; closed arrowhead) shows where a tube was detected at $\approx 10 \%$ below the optimal threshold of correlation.
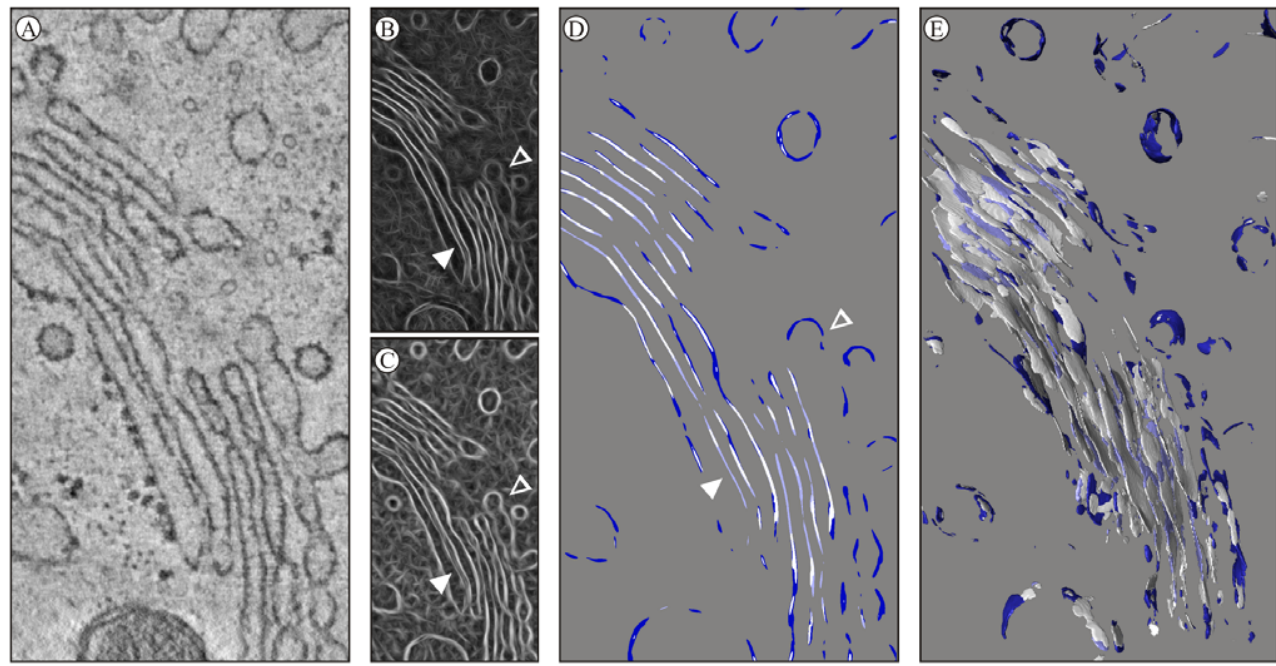

Fig. 5. Localization and annotation of the Golgi-apparatus. A small region of a tomogram containing a Golgi-apparatus is shown in (A). Cross-correlation results are shown for a cuboid model of a four voxel thick membrane matched both with a cuboid mask (B) and a spherical mask (C). Curved membranes are better detected using a spherical mask (open arrowheads), while the cuboid mask allows a clear distinction between membranes in close proximity to each other (closed arrowheads). An overlay of both cross-correlations exceeding the optimal threshold of correlation is shown in (D), where dark blue represents the area exclusively detected by using the spherical mask, light blue is the area detected only with the cuboid mask, and white is the overlapping area detected by both. (E) Shows a semi-automatic 3D-model of a Golgi-apparatus created by combining the cross-correlation results of both calculations. 
scale, microtubules appear as rather homogeneous structures that can be annotated quite effectively, membranous structures are often more complex to contour. To evaluate whether template matching can be used to visualize such membrane-systems we looked at the endoplasmic reticulum (ER) and the Golgi-apparatus. Both these organelles play an important role in the secretory pathway, and their transport mechanisms have been extensively studied. In recent years, electron tomography has been extremely valuable in understanding the architecture of these organelles. As a model for the membrane we used a cuboid template of four voxels thick with a corresponding cuboid mask of eight voxels thick; resembling the average thickness of the membranes manually observed in the tomogram. In Fig. 5 it is shown that automatic segmentation of complex membranous structures with a limited curvature is indeed possible using template matching with these settings (Fig. 5A, B, and D (light blue and white)).

Though large parts of the membranes are correctly annotated, a significant amount is missed (false negatives). Due to the cuboid shape of the template, regions in the ER where ribosomes are attached to the membranes were largely excluded. Furthermore, in the trans-Golgi network (TGN) the strong curvature and many membrane constrictions hinder automatic segmentation using this template and mask (Fig. 5B, open arrowhead). We found that by using a spherical mask (instead of a cuboid mask) that the curvature of the membrane resulted in less false negatives (Fig. 5C, open arrowhead, d (dark blue and white)). This is due to the fact that with the cuboid-shaped mask the background intensities are excluded. By including these with the spherical mask, the relative importance of the intensities describing the shape of the structure decreases. As a consequence, slightly curved membranes will still be considered to resemble the template as long as its environment (contained within the masked area) is devoid of any other structures. On the other hand, two membranes close together will be lost from the matching with a spherical mask (Fig. 5C, closed arrowhead), whereas the cuboid mask detected two clear distinct membranes (Fig. 5B, closed arrowhead). By combining the models created using both masks (Fig. 5D), we optimized the performance of annotation and were able to obtain a 3D surface-rendered representation of these membranous cellular compartments in an semi-automatic manner (Fig. 5E). The matchings were combined, by including those voxels that exceeded the optimal threshold of correlation for either or both of the conditions.

\subsection{Automatic detection of coated membrane domains on endosomes}

Cellular membranes have a heterogeneous composition. Differences in lipid and protein composition are associated with the formation of domains (Brown and London, 1997; Pfeffer, 2003), many of which are known to play a functional role in the cell. Among those, clathrin coats on endosomes (Raposo et al., 2001; Sachse et al., 2002) are suitable to evaluate the possibilities of using template matching to identify such domains. Endosomes possess a relatively simple membranous organization, and in electron tomographic reconstructions these coats can be visualised against the endosomal membrane as thick patches (Murk et al., 2003). To discriminate between these thick patches and the thinner uncoated membranes, we applied two artificially generated cuboid templates of distinct different thicknesses (six voxels and 16 voxels; Fig. 1E and J) to a tomogram of an endosome with clathrin coats. As shown in Fig. 6 (a and b, closed arrowheads), it is indeed possible to discriminate between coated and non-coated membranes. By comparing the automatically annotated model to the one published by Murk et al., we note that at a cross-correlation-threshold of 0.5 (the optimal threshold for the six voxels thick template)
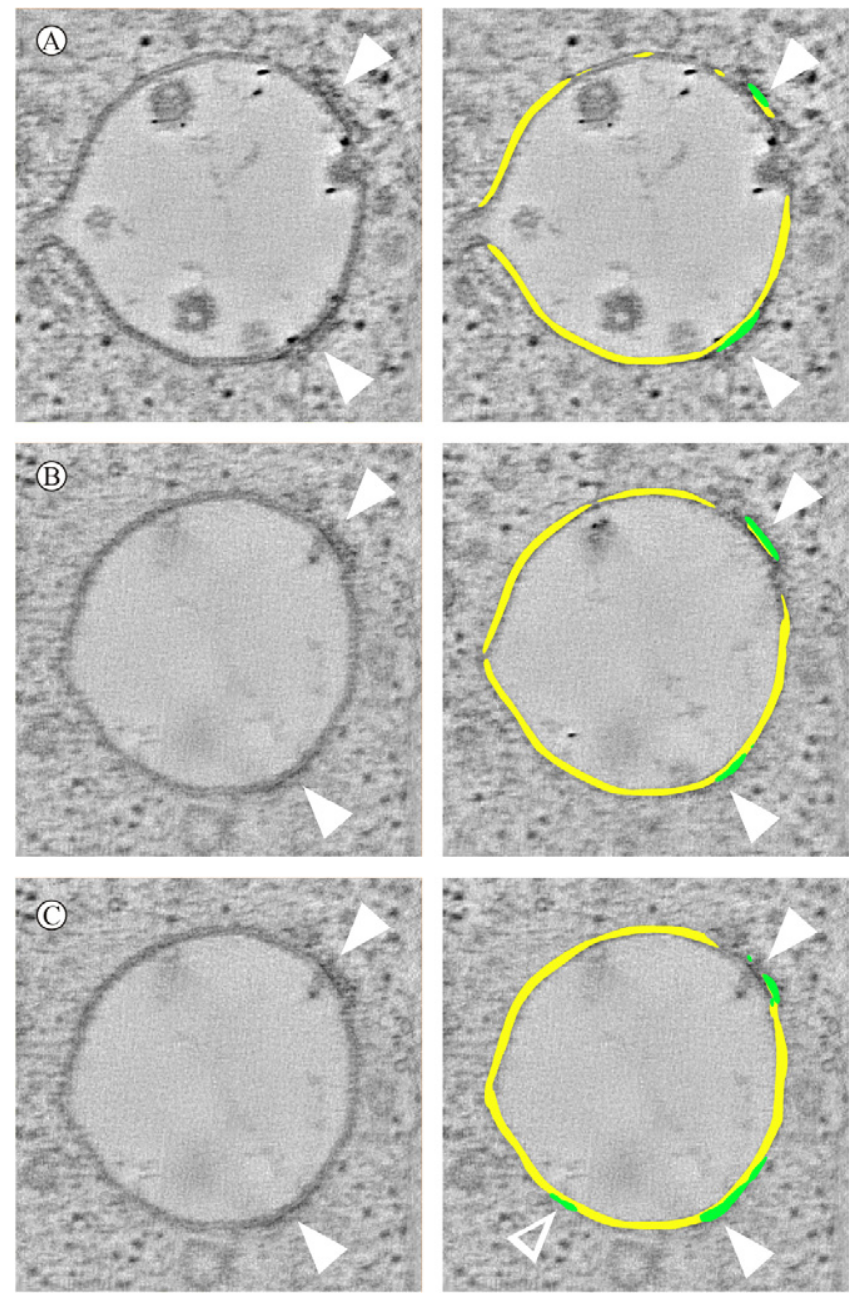

Fig. 6. Localization and annotation of thick clathrin coats on endosomes. We localized clathrin coats (green, close arrowheads) present on endosomal membranes (yellow) based upon a difference in stained thickness between coated and uncoated membrane, (A) and (B) show different optical slices with an overlay of the matched cuboid of 6 voxels (yellow) and 16 voxels (green) in thickness at a cross correlation threshold of 0.5 . (C) shows the same section as (B) but with a threshold of 0.45 . A region in the uncoated membrane appears (open arrowhead), where the thicker template has a higher correlation-value to the tomogram than in the surrounding membranes. 
all the manually identified, thicker clathrin coats were exclusively localized in the membrane. We noticed that when we lowered the threshold to 0.45 , a third region appeared in an area of uncoated membranes (Fig. 6C, open arrowhead). This value was considered the optimal threshold of correlation for the 16 voxels thick template.

Considering the resolution-range aimed for to annotate the cellular architecture, the template chosen to detect the clathrin patches was not based on the ultra-structure of clathrin, but based on the fact that clathrin coats simply appear thicker on stained membranes. As such, the technique is used as a tool to detect variations in membranestaining thickness instead of localizing specifically clathrin.

Heterogeneity within non-coated membranes of endosomes is not unexpected, since the membranes of internal vesicles in multi-vesicular bodies and its limiting membrane have different protein and lipid compositions (Kobayashi et al., 1998), indicating lipid sorting in the membrane. We, therefore, set forth to visualize membrane regions of different staining thicknesses (Fig.7A). We created several cuboid templates of different even thicknesses ranging from 4 to 18 voxels (Fig. 1D-K). If the membranes were homogeneous, and no detectable different domains would exist, each template is expected to fit equally well on every location of the membrane. Also, a single template - with a thickness closest to the thickness of the stained membrane - is expected to fit best for the entire membrane of the structure. However, in case of staining-heterogeneity, different templates will fit best at different locations corresponding to thicker and thinner stained-patches, and templates will not fit equally throughout the membrane. For each of the eight different membrane templates we set the threshold for the minimal cross-correlation to 0.5 (the highest optimal threshold for any of the templates). Those regions exceeding the threshold are presented in Fig. 7A. To visualize the distribution of thicker (green) and thinner (yellow) regions on the endosomal membrane we projected these regions on top of each other (Fig. 7B). While the thick, coated patches are clearly visible, colour variations (representing differences in stained thickness) can be
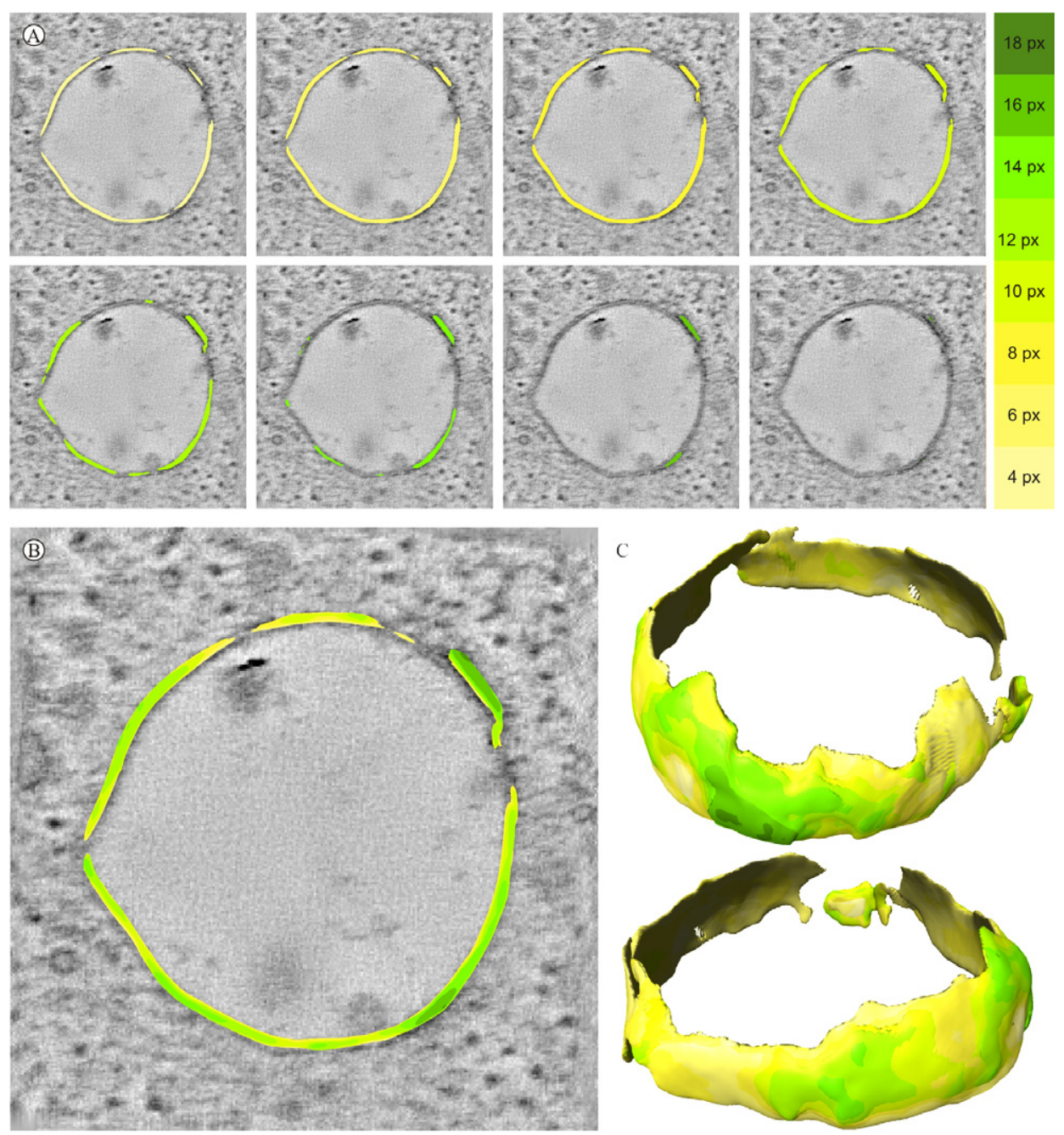

Fig. 7. Visualization of heterogeneity in stained membranes of a endosomal structure. A series of cuboid templates of increasing thickness (Fig. 1C-K) were matched to the endosome. Based on the local stained thickness of the membrane these templates matched differently as visualized in (A) separately for each template at a correlation threshold of 0.5 . In the overlay (B) and the 3D model (C) clear thinner and thicker stained regions can be seen indicating heterogeneity in membrane composition represented by a difference in membrane staining. 
observed throughout the regions of uncoated membrane. In Fig. 7C this heterogeneity in membrane staining is presented in 3D. It seems that these variations are not randomly distributed, since patches of connected regions are visible rather than randomly distributed small spots. This is an indication that these regions are genuine reflections of biological structures with different biological functions. It is however unclear at this time what they truly represent, and if the visualized heterogeneity has any biological significance in terms of protein sorting or vesicle budding.

\section{Conclusions}

For structures in tomograms of stained sections, template matching provides a less subjective way of analysing the data when compared to manual annotation. Template matching introduces a degree of quantification and automation in analysing morphological studies. Furthermore, it can help pinpoint to biologically relevant phenomena in the tomogram that are easily missed by eye alone. By varying a single property of an artificial (computationally generated) template matched to a volume, it is possible to examine this property by analysing how well each variation fits at a given location. We have illustrated this possibility by matching cuboid templates of increasing thickness to stained membranes to visualize patches of clathrin-coats and heterogeneity.

Template matching can be a valuable addition to manual annotation of tomograms of microtubules and membranous structures. Though it is possible to trace cellular structures automatically, the technique still requires a human component. First, if present, incorrectly annotated structures (false positives), have to be removed. This can be done either by raising the correlation threshold, or by manually erasing the falsely annotated structures. Second, if present, missed structures (false negatives) have to be compensated for, either by lowering the correlation threshold, or by manual contouring.

While template matching can visualize membranes in a cell, it cannot distinguish between membranes of different organelles. To achieve exclusive segmentation of the organelle of interest, correctly identified membranes of surrounding organelles have to be removed by manual intervention. Moreover, in some studies, different membranes of a single organelle (for example the different stacks of a Golgi-apparatus) have to be distinguished by user expertise. Such a distinction cannot be achieved by an automated template matching procedure.

To understand the overwhelming amount of structural details within a tomogram, segmentation steps are unavoidable. Template matching is one of the possible approaches to model the content of tomograms, and can help unravel complex cellular architecture in stained sections.

\section{Acknowledgments}

We thank Jean-Luc Murk, Hezder van Nispen tot Pannerden, and Hans Geuze for providing us with the tomo- grams of the endosome and its accompanying model. Also, David Lutje Hulsik, Alevthyna Yakushevska, Hakan Yakali, and Sandra Waaijenborg for their constructive discussions. Furthermore, Friedrich Förster, Reiner Hegerl, Stephan Nickell, Achilleas Frangakis, and Wolfgang Baumeister from the Max-Planck-Institut für Biochemie in Martinsried for providing the OmniMatch-software for template matching and, Bart Heupers from the SARA computing and networking services in Amsterdam for his help with integrating and running the OmniMatch-software on the Dutch grid environment. This research has been made possible by the Dutch IOP genomics project IGE3012.

\section{References}

Bennett, P.M., 1974. Decrease in section thickness on exposure to the electron beam; the use of tilted sections in estimating the amount of shrinkage. J. Cell Sci. 15, 693-701.

Böhm, J., Frangakis, A.S., Hegerl, R., Nickell, S., Typke, D., Baumeister, W., 2000. Toward detecting and identifying macromolecules in a cellular context: template matching applied to electron tomograms. Proc. Natl. Acad. Sci. USA 97, 14245-14250.

Brown, D.A., London, E., 1997. Structure of detergent-resistant membrane domains: does phase separation occur in biological membranes? Biochem. Biophys. Res. Commun. 240, 1-7.

Egerton, R.F., Li, P., Malac, M., 2004. Radiation damage in the TEM and SEM. Micron 35, 399-409.

Frangakis, A.S., Böhm, J., Förster, F., Nickell, S., Nicastro, D., Typke, D., Hegerl, R., Baumeister, W., 2002. Identification of macromolecular complexes in cryoelectron tomograms of phantom cells. Proc. Natl. Acad. Sci. USA 99, 14153-14158.

Frangakis, A.S., Hegerl, R., 2001. Noise reduction in electron tomographic reconstructions using nonlinear anisotropic diffusion. J. Struct. Biol. 135, 239-250.

Frangakis, A.S., Stoschek, A., Hegerl, R., 2001. Wavelet transform filtering and nonlinear anisotropic diffusion assessed for signal reconstruction performance on multidimensional biomedical data. IEEE Trans. Biomed. Eng. 48, 213-222.

Frey, T.G., Perkins, G.A., Ellisman, M.H., 2006. Electron tomography of membrane-bound cellular organelles. Annu. Rev. Biophys. Biomol. Struct. 35, 199-224.

Killian, J.A., 1998. Hydrophobic mismatch between proteins and lipids in membranes. Biochim. Biophys. Acta 1376, 401-415.

Kobayashi, T., Stang, E., Fang, K.S., De Moerloose, P., Parton, R.G., Gruenberg, J., 1998. A lipid associated with the antiphospholipid syndrome regulates endosome structure and function. Nature 392, 193-197.

Koster, A.J., Grimm, R., Typke, D., Hegerl, R., Stoschek, A., Walz, J., Baumeister, W., 1997. Perspectives of molecular and cellular electron tomography. J. Struct. Biol. 120, 276-308.

Kremer, J.R., Mastronarde, D.N., Mcintosh, J.R., 1996. Computer visualization of three-dimensional image data using IMOD. J. Struct. Biol. 116, 71-76.

Ladinsky, M.S., Mastronarde, D.N., Mcintosh, J.R., Howell, K.E., Staehelin, L.A., 1999. Golgi structure in three dimensions: functional insights from the normal rat kidney cell. J. Cell Biol. 144, 1135-1149.

Lučić, V., Förster, F., Baumeister, W., 2005. Structural studies by electron tomography: from cells to molecules. Annu. Rev. Biochem. 74, 833-865.

Mastronarde, D.N., 1997. Dual-axis tomography: an approach with alignment methods that preserve resolution. J. Struct. Biol. 120, 343-352.

Mcewen, B.F., Downing, K.H., Glaeser, R.M., 1995. The relevance of dosefractionation in tomography of radiation-sensitive specimens. Ultramicroscopy 60, 357-373.

Mogelsvang, S., Marsh, B.J., Ladinsky, M.S., Howell, K.E., 2004. Predicting function from structure: $3 \mathrm{D}$ structure studies of the mammalian Golgi complex. Traffic 5, 338-345. 
Moreau, P., Cassagne, C., 1994. Phospholipid trafficking and membrane biogenesis. Biochim. Biophys. Acta 1197, 257-290.

Moss, W.C., Haase, S., Lyle, J.M., Agard, D.A., Sedat, J.W., 2005. A novel $3 \mathrm{D}$ wavelet-based filter for visualizing features in noisy biological data. J. Microsc. 219, 43-49.

Murk, J.L., Humbel, B.M., Ziese, U., Griffith, J.M., Posthuma, G., Slot, J.W., Koster, A.J., Verkleij, A.J., Geuze, H.J., Kleijmeer, M.J., 2003. Endosomal compartmentalization in three dimensions: implications for membrane fusion. Proc. Natl. Acad. Sci. USA 100, 13332-13337.

Murk, J.L., Lebbink, M.N., Humbel, B.M., Geerts, W.J., Griffith, J.M., Langenberg, D.M., Verreck, F.A., Verkleij, A.J., Koster, A.J., Geuze, H.J., Kleijmeer, M.J., 2004. 3-D Structure of multilaminar lysosomes in antigen presenting cells reveals trapping of MHC II on the internal membranes. Traffic 5, 936-945.

Nezil, F.A., Bloom, M., 1992. Combined influence of cholesterol and synthetic amphiphillic peptides upon bilayer thickness in model membranes. Biophys. J. 61, 1176-1183.

Nickell, S., Forster, F., Linaroudis, A., Net, W.D., Beck, F., Hegerl, R., Baumeister, W., Plitzko, J.M., 2005. TOM software toolbox: acquisition and analysis for electron tomography. J. Struct. Biol. 149, 227-234.

Penczek, P., Marko, M., Buttle, K., Frank, J., 1995. Double-tilt electron tomography. Ultramicroscopy $60,393-410$
Pfeffer, S., 2003. Membrane domains in the secretory and endocytic pathways. Cell 112, 507-517.

Raposo, G., Tenza, D., Murphy, D.M., Berson, J.F., Marks, M.S., 2001. Distinct protein sorting and localization to premelanosomes, melanosomes, and lysosomes in pigmented melanocytic cells. J. Cell Biol. 152, 809-824.

Sachse, M., Urbe, S., Oorschot, V., Strous, G.J., Klumperman, J., 2002. Bilayered clathrin coats on endosomal vacuoles are involved in protein sorting toward lysosomes. Mol. Biol. Cell 13, 1313-1328.

Simons, K., Ikonen, E., 1997. Functional rafts in cell membranes. Nature $387,569-572$

Smondyrev, A.M., Berkowitz, M.L., 1999. Structure of dipalmitoylphosphatidylcholine/cholesterol bilayer at low and high cholesterol concentrations: molecular dynamics simulation. Biophys. J. 77, 2075-2089.

Volkmann, N., 2002. A novel three-dimensional variant of the watershed transform for segmentation of electron density maps. J. Struct. Biol. 138, 123-129.

Yunghans, W.N., Keenan, T.W., Morre, D.J., 1970. Isolation of golgi apparatus from rat liver. 3. Lipid and protein composition. Exp. Mol. Pathol. 12, 36-45.

Zambrano, F., Fleischer, S., Fleischer, B., 1975. Lipid composition of the Golgi apparatus of rat kidney and liver in comparison with other subcellular organelles. Biochim. Biophys. Acta 380, 357-369. 